\title{
Variations
}

Variations

Revue internationale de théorie critique

$17 \mid 2012$

Critique du travail

\section{Le mode artistique de la Révolution : de la gentrification à l'occupation (II)}

\section{Martha Rosler}

Traducteur : Lucia Sagradini

\section{OpenEdition}

Journals

Édition électronique

URL : http://journals.openedition.org/variations/430

DOI : 10.4000/variations.430

ISSN : 1968-3960

Éditeur

Les amis de Variations

\section{Référence électronique}

Martha Rosler, "Le mode artistique de la Révolution : de la gentrification à l'occupation (II) », Variations [En ligne], 17 | 2012, mis en ligne le 15 octobre 2012, consulté le 20 avril 2019. URL : http:// journals.openedition.org/variations/430 ; DOI : 10.4000/variations.430

Ce document a été généré automatiquement le 20 avril 2019.

Les ami•e•s de Variations 


\title{
Le mode artistique de la Révolution: de la gentrification à l'occupation (II)
}

\author{
Martha Rosler \\ Traduction : Lucia Sagradini
}

\section{NOTE DE L'ÉDITEUR}

Cet article est la suite de « Le mode artistique de la Révolution : de la gentrification à

l'occupation (I) » publié dans le précédant numéro de Variations : http://

variations.revues.org/206.

1 Cinquante ans après le plan Master de l'Université de Californie, comme Kerr l'avait prévu, la plupart des productions post-fordistes s'inscrivent désormais dans le champ de l'industrie de la connaissance. Tous les produits culturels, ainsi aplatis, deviennent désormais de l'« information ", écrasant les produits de l'écriture, de la recherche, du divertissement, tout ce que vous voudrez, et bien sûr, l'art. L'apparence et le mode de vie, qui en découlent, s'appuient en grande partie sur les notions de vertu et de vie séculière saine, telles qu'elles sont vendues à une génération élevée à l'école et aux campagnes médiatiques centrées sur les appels à une morale civique illustrée par « Il suffit de dire non à la drogue », «Le tabac tue » et « Sauvez la planète ! "; mais qui promeut néanmoins dans le même temps l'utilisation de produits pharmaceutiques. Ce way of life est alors plus susceptible d'être adopté par l'art urbain des écoles certifiées et par les travailleurs de l'industrie de l'art. Ce sont des jeunes professionnels urbains, peut-être, mais il ne s'agit plus des « yuppies » du passé (même si je suis curieuse de savoir ce que ce terme recouvre vraiment), c'est-à-dire qu'il ne s'agit pas d'avocats aux revenus élevés, de grands chefs d'agences de pub et d'éditeurs de magazines mais de travailleurs de la strate inférieure ayant des prétentions dans leur domaine. La vie de la cité sollicite les membres de ces industries ; elle est constituée de réseaux de petites boutiques où l'on bénéficie de relations de face-à-face et de tous les attraits de la vie urbaine. 
2 Cette préférence pour la ville peut être retracée à partir de l'essor de la croissance d'après-guerre dans les démocraties industrielles occidentales. Aux Etats-Unis, cet essor a conduit à l'accroissement de la prospérité de la classe moyenne et aussi à la migration de nombreux citadins vers les petites villes et les nouvelles zones pavillonnaires construites (bien que le racisme et les préjugés de classe aient été aussi d'importants facteurs de motivation), ainsi qu'à la consolidation des communautés. Le premier effet de l'exode urbain par la classe moyenne a été de provoquer la désertification de quartiers commerciaux, de magasins et d'industries, ainsi que l'évacuation de nombreux centres d'affaires des villes. Mais l'étape suivante a été le renversement de la tendance à aller en banlieue par les enfants ennuyés de la classe moyenne et par d'autres, comme les chefs d'entreprises et les yuppies tant redoutés. Ils y sont revenus, attirés par les plaisirs de la vie organisée en ville, comme les musées et les théâtres, ainsi que le mélange vertigineux d'anonymat, de communauté, et de diversité, et des potentialités que recèle l'imaginaire urbain. Pour souligner l'évidence, l'abrutissement, l'expérience totalement homogène de la vie dans les zones pavillonnaires, avec ses centres commerciaux identiques et ses fastfood. Cette vie périphérique n'offre pas la créativité désirante d'un appel au futur, et dans la mesure où le local existe encore aujourd'hui, c'est dans les villes ou les petites villes rurales, mais certainement pas dans l'univers clôturé des zones péri-urbaines que l'on retrouve cette potentialité.

3 Cette repopulation et cette transformation des villes se sont opérées en pas moins d'une génération. On est passé de villes avec des espaces dépourvus de commerces et d'entreprises, manquant cruellement de ressources, habitées par des gens pauvres, par la classe ouvrière et par des squatteurs vivant dans des habitations mal entretenues, à des villes réservée à une classe moyenne désirante, avec des espaces de divertissement et de shopping haut de gamme. Par les efforts concertés de ces métropoles, quelques-unes, chanceuses, sont devenues, parfois, des centres de la finance, de l'immobilier et de la gouvernance d'entreprise, ou de la fabrication dans des domaines tels que la pharmaceutique ou le haut lieu des industries des nouvelles hautes technologies. Après le déclin de l'épidémie de crack et cocaïne, qui a dévasté les communautés urbaines pauvres dans les années 1980, la police ne pouvait guère continuer à se concentrer sur «les fenêtres cassées ", à poursuivre la petite délinquance, selon la théorie que tout trouble visuel crée un sentiment de danger et encourage en outre le crime. Cette théorie est parfois vendue sous la rubrique de la tolérance zéro, un slogan sans substance. Agiter un climat d'agressions, non seulement exacerbe les tensions raciales et celles de classe mais éloigne en plus ceux-là mêmes que les villes souhaitent attirer. 
Les quartiers de Soho et d'East Village de New York ont prouvé à la fin des années 1970 que la question de la transformation d'anciens entrepôts et d'immeubles pourris en biens

immobiliers de grande valeur pouvait être accompli en permettant aux artistes de s'y installer. Si rien d'autre ne se présentait, alors l'intendance de la ville pouvait reconnaître ou s'identifier avec ces personnes et comprendre leurs besoins. Ces élus qui, à une époque, avaient pu soutenir le mouvement syndical, ont constaté que de telles forces avaient disparu. Les artistes, de plus, n'allaient pas s'organiser et rendre la vie difficile aux autorités municipales. Le modèle de Soho est devenu paradigmatique pour les villes du monde entier. Attirer de nouvelles petites industries, principalement celles de la haute technologie, a été une autre tactique répandue. Mais comment sommes-nous arrivés à la classe créative elle-même, la même partout autour du monde ? Peu importe à quel point

les arts (que ce soit les arts du spectacle ou les arts visuels institutionnalisés dans les musées) peuvent être vu, dans certaines villes, comme moteur économique, et même si cette condition ne s'applique évidemment pas partout : une nouvelle théorie urbaine était née. Entrez dans la politique du style de vie ${ }^{1}$.

La théorie de l'utilité civique des jeunes, éduqués mais souvent économiquement en marge, a d'abord été popularisée par un petit professeur peu connu et peu doué de la planification urbaine dans la Rust Belt de Pittsburgh, à Carnegie-Mellon University, un établissement portant le nom d'un industriel et d'un banquier. Ce que le professeur Richard Florida voyait autour de lui était des quartiers rendus plus douillets grâce aux efforts des récents diplômés, peut-être aussi par la mise en place de cafés et de petites entreprises dans les zones à faible loyer. L'environnement convivial pour le consommateur - respectueux des clients de la classe moyenne - accentue les goûts partagés transmis, depuis le milieu des années 1960, par le biais des écoles, de la musique, des films et des magazines. Ces goûts construisent aussi la place particulière où se nicher parmi les gens instruits, les professionnels de la classe moyenne. Les éléments de ce qui pourrait paradoxalement être reçu comme une vertu péri-urbaine (peut-être secourue par les images d'un style de vie nostalgique fourni par des magazines de mode et tiré des traditions des petites villes paradisiaques) allant du recyclage au jardinage, des arts à l'artisanat, étaient désormais ramenés au sein des quartiers délabrés de la ville.

La théorie de Florida consistait à vendre ces collections de jeunes gens, en général sousemployés, entrant dans ces catégories de sous-cultures, comme peuvent l'être aussi les homosexuels, et qui ont également eu tendance à se concentrer dans ce que l'on appelle « les quartiers bohèmes ", devenant pour les urbanistes un remède infaillible pour les zones urbaines tombées en désuétude. Son livre, The Rise of the Creative Class: And How It's Transforming Work, Leisure, Community, and Everyday

fut un « hit », un succès pour évangéliser le monde des affaires. Il jetait une bouée de sauvetage pour les gestionnaires municipaux souvent désespérés

. Créant ce qui semblait être des analyses systématiques, Florida a fait fructifier sa contribution dans un nouvel emploi et une nouvelle carrière de consultant. Il est maintenant à la tête du Martin Prosperity Institute de l'Université de Toronto et il est consultant pour les villes, les entreprises et organismes sans but lucratif à travers le monde. La prospérité, tout comme le joli nom de Florida, est l'un des mots-clé. Son site web dit : 
Le Groupe Classe Créative est une boutique de services consultatifs d'un cabinet composé d'éminents chercheurs, spécialistes de la communication et de conseillers en affaires. GCC combine une approche novatrice du leadership mondial et des stratégies éprouvées, offrant à ses clients dans le monde entier une intelligence critique du marché pour plus de compétitivité et une plus grande prospérité économique.

7 Les analyses de Florida s'accordent alors avec les gestionnaires municipaux tout en paraissant promouvoir la diversité d'une manière qui, en réalité, reproduit souvent ce qui est déjà en place. Mais ses prescriptions reposent également sur un tour de passe-passe. Beaucoup de ceux qui ont analysé les données utilisées, indiquent qu'il s'appuie sur des groupes de données standardisées recueillies lors de recensements. Il absorbe dans la classe créative tous les travailleurs de l'industrie de la connaissance : ceux des centres d'appels jusqu'aux analystes professionnels, aux scientifiques et aux mathématiciens, et à peine, les artistes. Mais il inclut ingénieusement dans son mixte un groupe statistiquement faible - les Bohêmes, qui comprend aussi les gays. L'économiste de Harvard, Edward Glaeser, a souligné à contrecoeur que ces données régressives suggèrent que la population gay aide au développement économique dans deux villes seulement (eh oui, dans l'Etat de Floride [Florida]...).

Ainsi, le vocable « créatif » a déferlé généreusement sur tout un chacun dans les industries de la connaissance. La convivialité attachée aux consommateurs et la couleur locale des types urbains - touchant un petit groupe relativement pauvre- devient alors le visage de l'autre, plus grand, plus riche, mais au fond, invisible, à savoir les membres du « noyau super créatif » de la catégorisation de Florida. Dans son jeu de passe-passe, les créatifs deviennent alors des personnes dont l'engagement est au cœur de leur travail mais aussi des personnes qui savent bien vivre, avec peu de moyens, mais beaucoup de goût, et c'est également une catégorie économique à cibler. Mais peu importe : Florida se

\section{leader d'opinion}

désigne lui-même comme un

, et donc comme un bonimenteur cognitif. Soigneusement élaborée son auto-description

confirme sa position au sommet de la hiérarchie des travailleurs de la classe créative,

l'arbitre de nos mésaventures

3. Les analyses de Florida sont des outils degestion

, des cadres rhétoriques qui font progresser la carrière des autres candidats de la classe des arbitres, les poussant à faire preuve de créativité, candidats qui les ramassent et les

brandissent, et peu importe le message qu'ils délivrent effectivement. D'une part, ils favorisent une sorte de vie en ville agréable dans les espaces publics qu'il est difficile de désavouer : verte, artisanale, conviviale, faisant du vélo une reine, sans doute en toute sécurité, et pour une foule de gens qui vivent aujourd'hui une sorte de rêve hippie peace-love-rock\&roll.

Mais avec ce rêve renaît une illusion collective de la classe moyenne, celle de rêver tous ensemble. Incommodes, mal accoutrés, les gens de la classe ouvrière sont marginalisés, poussés au loin, aux marges de la ville ou en lointaine banlieue. Dans l'enceinte de la ville, devenant forteresse, sont nouvellement mises en valeur les prédilections bourgeoises, égocentriques, / axées sur les rituels marchands et médiatisants qui accompagnent chaque étape de la vie, de la naissance aux enterrements de vie de garçon et de jeune fille, les mariages, les réceptions-cadeaux pour bébé, les naissances, les communions et même peut-être les décès. 
Le fait que, les analyses de Florida ne soient pas centrées sur les artistes et le complexe des institutions associées à l'art, ne dérange pas non plus les villes qui adoptent ses méthodes ni les destinataires de l'attention et de la générosité des projets urbains. De nombreux urbanistes ont fait remarquer que, pour les villes, soutenir les infrastructures et l'éducation conduit en réalité à la transformation urbaine et au bien-être des citoyens. Mais évoquer l'art apparaît souvent en soi comme vertueux ... bien que cela ne soit pas le cas. En Europe occidentale, l'art a été pris en charge par les

Etats comme un bien social, une partie commune de la collectivité. Nous pouvons aujourd'hui voir le piège de cela aux Pays-Bas, où la virulente droite populiste, comme elle est encline à le faire, met en scène l'art comme un hobby de gauche, un dangereux passe-temps menaçant les ressources de circonscriptions qui les méritent : à savoir le plus souvent celles des xénophobes ayant une peur terrible de la différence culturelle et de la perte de leur emploi

. Les personnalités publiques pro-art sont incapables d'argumenter avec succès contre les populistes anti-art, qui alignent une fois de plus le ressentiment

de la vieille petite bourgeoisie agrégé à des éléments pris à la classe ouvrière contre la nouvelle petite bourgeoisie des travailleurs de la connaissance - peut-être que le terme technique de manipulateur symbolique est plus adéquat -, et la classe technocratique des experts dont les produits de leur travail sont invisibles mais leurs résultats pernicieux, un groupe qui d'une manière ou d'une autre se reconnaît dans le culte commun et l'inutilité apparente de l'art et des artistes vivants et en dessinent le visage emblématique. La re-création des villes au profit de la classe moyenne, y compris de ses élites managériales et financières, sur le dos des jeunes, privés en bonne partie de leurs droits, doctorants et étudiants en art, semble aux élites de nombreuses villes et de nombreux pays, comme un compromis raisonnable. Celui-ci est généralement moins coûteux que le financement des écoles d'art ou des musées. Socialiser les pertes, prolétariser les travailleurs et privatiser les profits. Aux Pays-Bas, la théorie de Florida de la classe créative reste très populaire, et je doute que beaucoup de ses promoteurs municipaux définissent la classe créative comme étant composée des artistes ou des gens des musées. Ils sont plus susceptibles de partir à la recherche de respectables bourgeois, qui paient des impôts, de ceux dont la richesse génère de l'information et des boutiques de design.

De nombreuses critiques échouent naïvement à considérer que Florida, comme Clark Kerr, doit être classé parmi les sociaux libéraux. Florida se dresse ainsi sur les barricades rhétoriques, s'entendant avec les patriciens, défendant le droit de la classe créative à faire un travail pour soi, avec peu ou pas de compensation, et soutenant aussi bien la tolérance que les subventions. Les libéraux, et même, la plupart du temps, les néo-libéraux, sont heureux de célébrer les artistes et, mieux, les "créatifs", ce groupe amorphe de brasseurs, boulangers, agriculteurs urbains et baristas, aussi longtemps que leurs festivals et leurs célébrations peuvent être payés par les banques, les sociétés et les fondations et que leurs efforts sont civiquement étiquetés.

Pour revenir aux Etats-Unis, mais pas seulement, il est nécessaire de souligner que les institutions de l'architecture et des arts sont très heureuses d'être emportées par la marée urbaine de la classe créative. Les institutions artistiques bénéficient ainsi de l'attention des organismes gouvernementaux, des fondations, et ainsi de suite, mais les coûts sont également à considérer. Des artistes, déjà complices, consciemment ou 
inconsciemment, dans la renégociation urbaine par les élites, sont aussi devenus les acolytes de la gestion sociale. Les concessions immobilières ont longtemps été étendues aux artistes et à de petites organisations à but non lucratif, dans l'espoir d'améliorer leur attractivité et de les ramener ensuite vers des loyers haut de gamme. L'importance de l'art et du "penchant esthétisant» permet ainsi aux musées et aux groupes d'architectures, ainsi qu'aux groupes d'artistes (artistes et administrateurs des arts de petites organisations à but non lucratif) de se glisser dans la conversation sur la branchitude citadine.

Désormais, les instituts culturels d'Architecture organisent des réunions et publient des bulletins d'information vantant les villes « vivables ». Alors que les jeunes conservateurs s'efforcent de devenir les imprésarios de " pratiques sociales ». Les écoles sont progressivement devenues les gestionnaires et les faiseurs du développement artistique.

D'une part, par la préparation des artistes à entrer dans le marché de l'art, d'autre part par l'injection de restrictions disciplinaires sur un art fabriqué comme une arme de gouvernement, en instituant des départements de «pratique publique » et de " pratique sociale », des séminaires de "nouvelles formes de militantisme, pratique communautaire, organisation alternative et le leadership participatif dans les arts » qui explorent « les liens multiples entre l'art et

la société afin d'examiner les moyens par lesquels les artistes (...) s'engagent sur des questions civiques et font entendre leur voix dans le domaine public 5. 》

George Yúdice, dans son livre,The Expediency of Culture: Uses of Culture in the Global Era , explore les usages de la culture en tant qu'élément de gouvernement et de la gouvernementalité, au sens de Foucault, tandis que les activistes mobilisent la culture au service des gens

${ }^{6}$. Yúdice, suit Fredric Jameson, lorsqu'il affirme que toutes les relations sociales ont été « culturalisées », et il s'associe à d'autres lorsqu'il fait remarquer que ce que nous appelons la pratique sociale fait de l'artiste un agent de l'Etat; bien plus, c'est la fin de l'avant-garde, extirpant les actions des artistes du domaine de la critique pour celui de l'amélioration. Il décrit un impératif d'

efficacité

découlant des pratiques des administrateurs d'art. En 1997, un rapport du gouvernement, intitulé

American Canvas,

a expliqué que les arts doivent désormais adopter une approche pragmatique, « traduisant la valeur des arts en termes de valeur générale, civique, sociale, et éducative » (...) « loin des fonctions traditionnelles esthétiques » visant à convaincre aussi bien le public que les élus eux-mêmes. Il conseille aux organismes artistiques de se joindre aux « districts scolaires, aux services des parcs et des loisirs, aux congrès et aux bureaux d'accueil, aux chambres de commerce et organismes de protection sociale (...) pour mettre en évidence les aspects utilitaires de l'art... » et de trouver un soutien grâce à «l'échange de biens et services (...) d'aborder les problèmes sociaux pour améliorer l'éducation en fournissant le "contenu" de l'autoroute de l'information nouvelle... » Les musées au cours des deux dernières décennies ont généralement obtenu des fonds pour augmenter leurs fonctions d'enseignement et de service en direction des pauvres, des exclus, et des non-élites, la classe supposément non-créative. Maintenant la portée de la mission s'est élargie à l'amélioration de la ville elle-même. Cette année, par exemple, le 
New Museum de New York a parrainé un «festival » de la ville, comportant la promotion de maires et de fonctionnaires, d'universitaires et d'experts de la politique urbaine, une pléthore d'associations à but non lucratif, et ainsi de suite, tandis que, à l'autre extrémité, un ambitieux monde de l'art, à but non lucratif lui aussi, dirige une conférence annuelle sur les pratiques publiques du monde entier. Tous deux ont recours à un langage ampoulé et à une culture de célébration pour souligner l'importance de leurs propres travaux.

Le luxueux fabricant automobile, BMW, a, de son côté, rejoint le Musée Guggenheim pour mettre sur pied « un laboratoire mobile pour inspirer des idées novatrices pour la vie urbaine », avec un artiste de grande envergure et les noms de grands architectes rattachés

7. Le « Lab » lie fermement la société, le musée,

l'architecture, l'art et le divertissement à l'embourgeoisement des villes. La citoyenneté urbaine a remplacé désormais les autres façons de se faire mousser pour les soi-disant citoyens d'entreprise. Soit dit en passant, ils aiment faire du vélo. Comme le fait Urban

Omnibus, qui aime aussi « l'art comme activateur urbain. »

L'Urban Omnibus est une plate-forme en ligne de la vénérable Architectural League de

8. Par exemple, le projet,Civic Action: A Vision for Long Island City

, décrit une nouvelle aventure, développée en collaboration avec deux musées d'art contemporain, qui « invite des équipes d'artistes à proposer des visions pour l'avenir de Long Island City ", un quartier faisant partie du Queens, à New York. Il s'agit de promouvoir une zone de ruine post-industrielle en un nouveau lieu haut de gamme résidentiel grâce au front de mer. Un autre projet,

Making Room, est « un projet de

recherche, de conception et de plaidoyer pour façonner le logement à New York et répondre aux besoins changeants de la façon dont nous vivons aujourd'hui

9. »

Il est désespérant de voir l'empressement visible des artistes à être enrôlés par les élites dirigeantes, trouvant une validation par le marché, mais aussi (selon mon interprétation) par les villes et les élites planificatrices et amatrices d'art, qui, j'imagine, a conduit Bifo à dire aux artistes et aux personnes intéressées de renoncer au terme d'artiste en faveur de celui, apparemment non marchandisé, de poète. Et en plus il n'est même pas français.

Mais il y a des moments où la professionnalisation de la formation artistique dans les collèges et les université et les efforts conjugués des curateurs pour annexer et transformer en marques les initiatives artistiques en cours ont l'avantage d'élargir les réseaux sociaux et le vocabulaire de l'action. La réception populaire de l'art et son public considérablement élargi ont alors pour effet de créer une visibilité mutuelle des artistes et des autres personnes ayant des sous-emplois qu'elles soient instruites ou pas. La tendance est peut-être celle de la professionnalisation et de l'embourgeoisement des artistes, ainsi que pour d'autres membres du secteur symbolique, mais là où l'avenir fissure le mur de briques, cela peut avoir des conséquences révolutionnaires. Il suffit de regarder autour de nous.

Dans son livre, George Yúdice soutient que, surtout dans les pays de la périphérie, les activistes peuvent utiliser la primauté de la culture pour organiser leurs communautés, comme je l'ai suggéré précédemment. Mais certains observateurs soulignent aussi la tendance de gauche des artistes et leur préoccupation sociale, y compris dans les économies avancées, sans avoir besoin de l'appui de bailleurs de fonds institutionnels ou 
de curateurs. Comme les autres participants des mouvements d'occupation qui se déroulent partout dans le monde, et comme ceux qui ont participé à des mouvements antérieurs, les artistes ont tendance à vouloir donner leur énergie et leurs capacités au mieux-être social et à la rêverie utopique. La sociologue Ann Markusen écrit ainsi :

Beaucoup de sociologues et de théoriciens affirment que les artistes servent de conscience à la société, en étant la source la plus plausible d'impitoyables critiques et le meilleur des soutiens aux impopulaires questions tels que la paix, l'environnement, la tolérance et la liberté d'expression.

La classe créative n'existe sans doute pas sous la forme dans laquelle Florida a établi ses frontières, et ce n'est peut-être pas tout à fait le sauveur social que Markusen suggère puisque les caractéristiques qu'elle impute aux artistes sont également les « causes » préférées de la classe créative. Mais dans un ralentissement économique prolongé, comme celui que nous traversons actuellement, il devient clair à quel point les artistes et les autres membres de la communauté artistique sont un secteur de la classe (ou non)nationale dont la composition est forgée et encline, comme l'exigent les clichés, à penser globalement et agir localement. Nous pouvons voir les activistes qui occupent revendiquer des droits, créer une présence, mettre en place un nouvel espace public, exigeant la réintégration de la politique en refusant de simplement présenter des demandes à des gouvernements représentatifs, mais plutôt choisissant d'adopter la démocratie, qui avait, depuis longtemps, fait partie de la culture américaine, mais seulement, combinée au double canon du néo-libéralisme. Tout en me félicitant de ce qui est nouveau, je ne peux m'empêcher de revenir au passé, non pas pour évoquer l'exigence d'auto-gouvernance qu'il y eut au XVIIIe siècle initiée par un groupe de bourgeois rebelles dans les colonies américaines mais pour rappeler l'American Civil Rights Movement et l'un de ses héritiers, le Free Speech, un mouvement étudiant des années 1960 qui connut un fort écho de par le monde, où la démocratie, c'est-à-dire la démocratie directe, sans représentation, était l'idée directrice, du moins le degré zéro du mouvement dans les premières années

10

. Dans cette itération particulière, les contributions d'artistes célèbres tels que Shepard Fairey (renommé grâce à son affiche de Obama/la campagne de l'Espoir de 2008) ont été accueillis poliment, mais il n'est pas difficile de voir, à côté, que les occupations sont elles aussi de grands travaux artistiques dans l'espace public réalisés par des milliers de participants ${ }^{11}$. Dans le cadre du groupe des diplômés, et, (paix

à Bifo), le grand nombre d'artistes - formant le noyau de l'armée urbaine des sous rémunérés, des non rémunérés et dont Florida souhaite exploiter les activités, ceux-là vivent dans un état de précarité qui peut les conduire à rechercher des solutions sociales nouvelles et inattendues. C'est là que le mode de la production artistique entre en jeu.

Le mode de production, nous nous en souvenons, comprend les forces de production mais aussi leurs relations, lorsque ces deux éléments sont en conflit, une crise naît. Si la thèse de la classe créative peut être vue comme une sorte d'hymne à l'harmonie entre les forces créatrices de la production et les relations sociales urbaines qui pourraient être mobilisées au bénéfice des villes dépourvues de capital industriel, peut-être alors, que les occupations actuelles par la base peuvent être considérées comme l'inévitable surgissement du conflit entre les créatifs et la ville qui les exploite. Il est intéressant à cet égard, que le cri de guerre ait été "Occupy» qui répond à l'injonction de Florida de "gentrify ». "Occupy", c'est ce cri qui cherche à occuper l'espace, d'une manière analogue aux mouvements radicaux antérieurs de quête de liberté dans l'émancipation, de 
république dans la démocratie, et d'égalité dans la justice. Florida dit " gentrify », nous, nous disons « occupy».

\section{NOTES}

1. Bien que la diabolisation de la classe ouvrière et des habitants pauvres des zones mûres pour la récolte de l'immobilier soit une tactique de longue date, les « gens bien » qui arrivent n'ont pu y accéder avec leur propre profil que récemment ; antérieurement le privilège de classe était considéré comme un acquis mérité.

2. New York, Basic Books, 2003. Florida n'a pas sorti de sa tête l'idée de classe créative mais il l'a popularisée avec des catégories statistiques. Selon sa thèse, la catégorie correspondant à la classe créative représente environ $30 \%$ des travailleurs américains, mais comme nous le verrons cidessous, les groupes qu'il utilise sont problématiques.

3. Christopher Dreher, entretien avec Richard Florida, Salon 7 Juin 2002. (h ttp://www.salon.com/2002/06/06/florida_22/) Richard Florida : (...) pour exploiter la créativité des fins économiques, vous avez besoin d'exploiter la créativité sous toutes ses formes. Vous ne pouvez pas générer une économie de technologie ou une économie de l'information ou une économie de la connaissance que si vous maîtrisez les aspects multidimensionnels de la créativité. (...) Il y a trois types de créativité : la créativité technologique (...), la créativité économique, (...) et de nouvelles industries et la créativité culturelle et artistique, (...) de nouvelles façons de penser les choses, de nouvelles formes d'art, de nouveaux designs, nouvelles photos, de nouveaux concepts. Ces trois aspects doivent se réunir pour stimuler la croissance économique. (...) La classe créative est composée de deux dimensions (...) les scientifiques, les ingénieurs, les gens des technologies, des artistes, des créatifs, des musiciens - soi-disant bohèmes qui sont environ $12 \%$ de la main-d'œuvre ... le cœur super créatif est vraiment la force motrice de la croissance économique. En plus du noyau super créatif, j'inclus les professionnels de la création et les gestionnaires, les avocats, les gens de la finance, les techniciens qui utilisent aussi leurs idées, la connaissance et la créativité dans leur travail. Je n'y inclus pas les gens des services ou des entreprises qui font usage de créativité dans leur travail. 
4. L'aile droite aux États-Unis peut se cacher sous une peau de mouton populiste, mais en réalité il s'agit d'un mouvement dirigé et créé par les élites financières. Il a lancé sa guerre contre l'art et les artistes dans les années 1980 dans le cadre d'une panique morale appelée par ses opposants les guerres culturelles. Des membres de la classe ouvrière, autrefois partisans du Parti Démocrate, ont rejoint les électeurs ruraux dans le giron républicain, entraînés par des appels au chauvinisme et aux soi-disant valeurs traditionnelles, tandis que le Parti Démocrate avait déjà renoncé à soutenir les travailleurs urbains et les pauvres. Cette campagne récurrente, souvent

désignée par l'usage en raccourci du slogan « Dieu, des armes, et des Gays », concentre une attaque contre les droits reproductifs et d'autres aspects de l'autonomie des femmes. Il n'y a pas, au départ, une attaque contre l'Islam, mais elle pourrait à juste titre être accusée

d'antisémitisme. L'attaque contre le financement de l'art et des institutions de l'art a pris la forme d'une défense d'icônes du christianisme et d'icônes chrétiennes alors même que les EtatsUnis avaient encore besoin de présenter la liberté artistique comme un emblème de son mode de vie démocratique ; dans les années 1980, cela signalait son opposition au bloc de l'Est. Mais il était mis en avant que l'argent du gouvernement ne pouvait pas être consacré à soutenir des attaques contre le pauvre christianisme assiégé. Bien que la campagne actuelle aux Pays-Bas, et peut-être dans le reste de l'Europe occidentale, maintienne une position contre la communauté LGBT et ne se préoccupe guère des droits des possesseurs d'armes, leurs appels patriotiques prenant une forme différente, leur axe à l'égard des femmes est inversé : il est centré sur un faux problème pour le droit des femmes en faisant surgir le visage d'un Islam menaçant. En Europe de l'Est, l'homophobie conserve souvent sa place dans la croisade morale.

5. Ces citations proviennent d'une annonce d'emploi diffusée par un service d'une grande université qui propose « une maîtrise en politique de l'art qui traite, dans une tonalité militante, le nexus entre la politique faite par l'art et la politique qui fait l'art. » En dépit de mon scepticisme, je ne veux pas rejeter le potentiel d'un tel réseau de formation et d'un tel programme. Le problème réside dans la courte durée de vie que de telles initiatives peuvent avoir avant que l'institution ne les réduisent en zombies.

6. The Expediency of Culture: Uses of Culture in the Global Era, Durham, N.C., Duke University Press,

2003.

7. Voir : http://www.bmwguggenheimlab.org/. Il y a eu une tentative infructueuse de la part des artistes d'occuper le laboratoire lors d'une journée d'actions des artistes.

8. Urban Omnibus est financé par Rockefeller Foundation's New York City Cultural Innovation Fund, le National Endowment for the Arts, le New York City Department of Cultural Affairs et le New York City Council.

9. La phrase, " how we live now ", met en évidence un ensemble prévisible d'hypothèses sur qui constitue ce «nous». 
10. Si on ne regarde pas seulement les meetings urbains dans les premiers jours des colonies américaines, on voit explicitement que la non-violence, la démocratie participative comme modèle d'organisation a été mis en avant par l'un des groupes centraux au mouvement des droits civiques, à savoir le Student Nonviolent Coordinating Committee, ou SNCC. Beaucoup de jeunes militants étudiants ont rejoint les campagnes de la SNCC Freedom Rider qui ont influencé les principes décrit peu après dans la Déclaration de Port Huron, un document fondateur $\mathrm{du}$ mouvement étudiant et anti-guerre. Naturellement, l'histoire, les origines et les influences de ces mouvements sont plus complexes que je ne peux l'esquisser ici. Le discours galvanisant du leader étudiant Savio Mario de Berkeley, comprend ce qui suit dans son préambule : «Je vous demande de considérer - s'il s'agit d'une entreprise, et si le Conseil des Gouverneurs est le Conseil

d'Administration, et si le président Kerr, en fait, est le gestionnaire, alors je peux vous dire quelque chose - la Faculté est faite d'un tas d'employés et nous sommes de la matière première ! Mais nous sommes un groupe de matières premières, ce qui ne veut pas dire être - et [d'] avoir tout pouvoir sur nous. Cela ne veut pas dire être transformé en n'importe quel produit !... Ni de finir par être achetés par certains clients de l'Université, qu'il s'agisse du gouvernement, qu'il s'agisse de l'industrie, ou de l'entreprise, parce qu'ils organisent l'emploi, ou que ce soit n'importe qui ! Nous sommes des êtres humains!» 11. Des groupes d'artistes abordent de plus en plus cette question, pour le meilleur et pour le pire ; voir par exemple :

http://newamericanpaintings.wordpress.com/2011/11/09/the-art-of-occupation/ et http:// www.villagevoice.com/2011-10-19/art/what-does-occupy-wall-street-mean-for-art/

\section{INDEX}

Mots-clés : critique du travail, correspondance, artistes, socialisme vert, travailleurs culturels, Wertkritik, Gorz André, dialogue, Vincent Jean-Marie, écologie, modèles alternatifs, Marx Karl, penser le Capital, ethos du travail, Eglise Orthodoxe, salariat, domination

\section{AUTEURS}

\section{MARTHA ROSLER}

Artiste new-yorkaise et iconoclaste mondialement connue, auteure d'essais qui s'inspirent de la Théorie critique, nourris de ses rencontres avec Herbert Marcuse ou Frederic Jameson. 